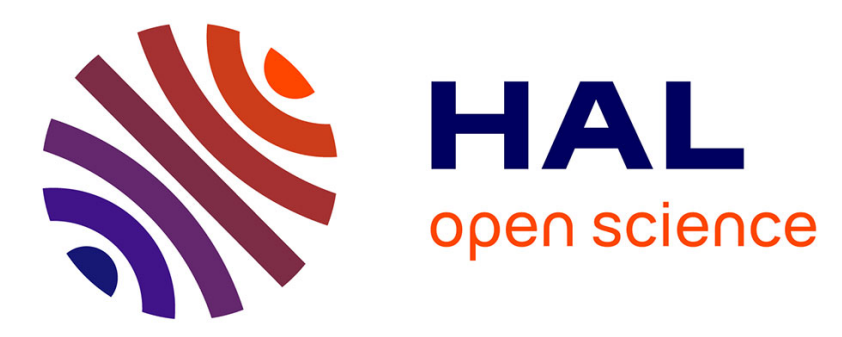

\title{
Modeling of Radiation-Induced Bystander Effect Using Monte Carlo Methods
}

J. Xia, L. Liu, J. Xue, Y. Wang, L. Wu

\section{To cite this version:}

J. Xia, L. Liu, J. Xue, Y. Wang, L. Wu. Modeling of Radiation-Induced Bystander Effect Using Monte Carlo Methods. The Seventh International Symposium on Swift Heavy Ions in Matter, Jun 2008, Lyon, France. hal-00216213

\section{HAL Id: hal-00216213 \\ https://hal.science/hal-00216213}

Submitted on 31 May 2008

HAL is a multi-disciplinary open access archive for the deposit and dissemination of scientific research documents, whether they are published or not. The documents may come from teaching and research institutions in France or abroad, or from public or private research centers.
L'archive ouverte pluridisciplinaire HAL, est destinée au dépôt et à la diffusion de documents scientifiques de niveau recherche, publiés ou non, émanant des établissements d'enseignement et de recherche français ou étrangers, des laboratoires publics ou privés. 
MODELING OF RADIATION-INDUCED BYSTANDER EFFECT USING MONTE CARLO METHODS

\author{
Junchao $\mathrm{Xia}^{+}$, Liteng $\mathrm{Liu}^{+}$, Jianming Xue *, Yugang Wang
}

State Key Laboratory of Nuclear Physics and Technology, School of physics, Peking University,

Beijing 100871, People's Republic of China

Lijun $\mathrm{Wu}$

Key Laboratory of Ion Beam Bioengineering, Institute of Plasma Physics, Chinese Academy of Sciences, Hefei 230031, People's Republic of China

\begin{abstract}
:
Experiments showed that the radiation induced bystander effect exists in cells, or tissues, or even biological organisms when irradiated with energetic ions or X-rays. In this paper, a Monte Carlo model is developed to study the mechanisms of bystander effect under the cells sparsely populated conditions. This model, based on our previous experiment which made the cells sparsely located in a round dish, focuses mainly on the spatial characteristics. The simulation results successfully reach the agreement with the experimental data. Moreover, other bystander effect experiment is also computed by this model and finally the model succeeds in predicting the results. The comparison of simulations with the experimental results indicates the feasibility of the model and the validity of some vital mechanisms assumed.
\end{abstract}

PACS: 87.50.-a; 61.80.Az; 87.53.Wz

Key words: Irradiation induced bystander effect, sparsely distributed cells, Monte Carlo simulation.

${ }^{+}$Junchao Xia and Liteng Liu contributed equally to this paper.

*Corresponding author: jmxue@pku.edu.cn 


\section{Introduction}

Studies in recent decades have proved that a radiobiological phenomenon named 'Radiation Induced Bystander Effect' (RIBE) could be observed with various endpoints ${ }^{[1,2]}$. Unlike traditional conditions in which the whole cell or organism was irradiated and damaged homogeneously and directly, RIBE represents a paradigm shift in our understanding of the radiobiological effects of ionizing radiation in which extra-nuclear and extra-cellular effects may also contribute to the final biological consequences $^{[3]}$. RIBE also implies that damages will occur in non-irradiated cells in response to the signals from neighboring irradiated cells.

Although RIBE has been admitted and verified by numerous experiments, the underlying mechanisms remain vague. For example, there are many experiments showing evidently that the level of RIBE is independent of the distance between the targeted cells and the bystander cells ${ }^{[4.5]}$. The mechanisms within, however, are still unknown.

So far, a few mathematical models, such as $\mathrm{BaD}^{[6]}$ and $\mathrm{BSDM}^{[7]}$, etc, have been established to explain and predict the experimental results. These models paid more attention on the modulation of parameters according to the experimental data than on the underlying biological mechanisms. Recently, a Monte Carlo method was developed to simulate the RIBE in sparse condition ${ }^{[8]}$ based on the experimental results ${ }^{[5]}$. The simulation, however, was unable to predict the approximately randomized distribution of damaged cells in the experiments until having introduced the "shaking effect". 
In the last few years, more experimental results have come out concerning the sort of the signaling molecules released from the irradiated cells and the signals' transmission and interaction with the bystander cells. One of the most recent results discovered that two possible bystander signaling factors, NO and TGF- $\beta 1$, are involved in RIBE ${ }^{[9]}$. Furthermore, these two signaling factors are interdependent: TGF- $\beta 1$ is considered to be the downstream product of radiation-induced $\mathrm{NO}$, while NO level will also be affected by TGF- $\beta 1$, which can be released from targeted cells and then diffuse freely in the medium due to its stability. A calcium-dependent pathway was proposed to be responsible for the inter-membrane transmission ${ }^{[9]}$.

This present simulation is also based on Monte Carlo method, aiming at studying how the bystander cells react in different distances from the cell irradiated in the center of the dish. The corresponding experimental data is from our previous experiment, performed in a cells sparely distributed situation ${ }^{[10]}$. As shown in the figure 1,130 cells were put in a round dish while the cell in the center was irradiated at 1Gy X-rays. The endpoint is survival. The results showed that, after three days' culture, there was no obvious difference in the magnitude of RIBE within the bystander cells at different distances to the irradiated cell in the center of the dish.

\section{Methods and models}

This simulation consists in the motion of the signals and their interaction with the cells under certain mechanisms. It is assumed that the original signals are released by the central cell irradiated directly. With the two dimension simplification, from 
Einstein Equation, the time interval $\Delta \mathrm{t}$ could be decided:

$$
\left\langle r^{2}\right\rangle=6 D \Delta t
$$

After each $\Delta \mathrm{t}$, the signal moves a distance accordingly and its direction is randomly selected. Here, $\boldsymbol{D}$ represents the translational mobility of the signal in the medium, which could be determined by the mass of the molecule ${ }^{[11]}$. Since TGF- $\beta 1$ could quite likely be the signaling molecule, $\boldsymbol{D}$ is chosen according to the mass of cytokine (TGF- $\beta 1$ is one member of cytokine), that is about $10 \mathrm{KDa}$. Thus, the signal's translational mobility is approximately $10^{8} \mathrm{~nm}^{2} \mathrm{~s}^{-1}$. Via $D$, the simulation time could be converted to the real time in the experiment, and the simulation time in this study represents 72 hours of real time as the experiment $\operatorname{did}^{[5,10]}$.

After each step, each signal will be examined by its position and accordingly cell damages will be determined. To describe the process more precisely, the following are the main assumptions during the determination in the previous model ${ }^{[8]}$ :

(1)A reaction between a signal and a bystander cell occurred when the distance between the signal and the cell center was less than a reaction radius $\boldsymbol{R}$. $\boldsymbol{R}$ was assumed to be $10 \mu \mathrm{m}$;

(2) whenever a reaction occurred, the signal was ruled out of the simulation, while the cell would be considered as damaged with probability $\boldsymbol{P}_{\text {dam }}$;

(3) bystander cells could in turn emit signals with probability $\boldsymbol{P}_{\boldsymbol{e}}$.

Based on the most recent experimental results, this present model focuses on the improvements on the above assumptions ${ }^{[8]}$. With introducing the first assumption completely into the model, however, there is an important correction as to the second 
assumption: when the signal hits the cell, if the cell is damaged, the signal will be ruled out from the system, i.e. absorbed by the cell. However, if the cell is not damaged, the signal should return to the medium and continue to move randomly in the medium instead of being absorbed. This amendment has a great influence on the macro distribution of damaged cells. In a biological sense, it means that it is possible for the signal (TGF- $\beta 1$ ) to be bound to the cell membrane and then induce a series of downstream intracellular reactions. Otherwise, it may leave the cell and keep moving randomly in the medium. As to the third one, the parameter $\boldsymbol{P}_{\boldsymbol{e}}$ is also introduced. Since the bystander cells have comparatively weak effects, it is assumed that the bystander cells will re-emit at most one signaling molecule to the medium if they do.

Furthermore, lifespan is added as a new parameter. Actually each signaling molecule disappears with time, i.e. the life time should be considered. In this simulation, a signal would be removed if its existing time exceeds the lifespan. Biologically speaking, this lifespan does not necessarily refer to the real life span of cytokine. It may be the activating time and its overall approximation may also include the possibility of the signal's bounding to the cell but without causing damages.

The percentage of naturally damaged cells is also included. It is proposed to obey a Gauss distribution:

$$
P_{\text {nat }}=\sqrt{\frac{1}{2 \pi \sigma}} \exp \left(-\frac{(x-\mu)^{2}}{2 \sigma^{2}}\right)
$$

$\mu$ and $\sigma$ represents the mean value and deviation of the distribution, respectively, which are both obtained from the data of the control groups in the experiment ${ }^{[10]}$. 


\section{Results}

In the simulation, the number of the original signals generated in the center of the dish is set to be 10,20 or 30 . For each original signal number, $\boldsymbol{P}_{\text {dam }}, \boldsymbol{P}_{\boldsymbol{e} m}$ and lifespan are adjusted manually to make the final distribution agree with the experimental data. Modulated parameters are shown in the table 1. The figure 2 shows the results including the comparison between the simulation and the experimental results

As the figure 2 demonstrates, for each set of parameters, the general trend of the ratio of damaged cells in different areas agrees well with the experimental data. The magnitude of bystander effect, i.e. the ratio of damaged cells in each area, is comparatively independent of the distance from the center. The first area has a relatively higher damage ratio, but generally keeping in the same level as those in farther areas. Even in the most distant district of the dish, the bystander effect still does not demonstrate obvious differences.

With the improvement of assumption (2), three sets of parameters are obtained agreeing with the experimental data. However, there are several differences among them. There should be other rational restrictions and observations, which are mentioned in the discussion

\section{Discussion}

Radiation Induced Bystander Effect (RIBE) has been discovered both in vitro and in vivo ${ }^{[1,2]}$. The gap junction intercellular communication (GJIC) was proved to play a significant role when physical contacts between neighboring cells are involved ${ }^{[12]}$. 
Furthermore, the ICM experiment suggested that there should be signaling molecules in the medium accounting for the transmission of the damage signal ${ }^{[13]}$.

This present model focuses on the intercellular transmitting process in cells sparsely populated conditions. As was mentioned above, the transmitting signal in the medium is TGF- $\beta 1$ most probably, which could be the downstream production of the intracellular reaction with the participation of $\mathrm{NO}$, and through a $\mathrm{Ca}^{2+}$ pathway probably. These results offered the basis for this model to renew the assumptions. This model improved some basic parts of the methodology of the previous Monte Carlo simulation: the signals would return to the medium instead of being ruled out when it fails to cause damages to the cell; another parameter, lifespan, is added in this model. Furthermore, the assumption for parameter $\boldsymbol{P}_{\boldsymbol{e}}$ is approved to be more biologically reasonable: after the signal TGF- $\beta 1$ leads to the production of NO by binding to the cell membrane, NO would in turn reproduce TGF- $\beta 1$, as a re-emitting production.

A manually adjusting process guarantees the agreement between the experimental data and the simulation results of each set of parameters. By comparing the parameters at different levels of the original signal number, the parameter changing trend could be observed. When the original number level is relatively high (num=20 or 30), $\boldsymbol{P}_{\text {dam }}$ is comparatively low to avoid making high damage ratio in the center area. Moreover, for the highest level of the original signal number, $\boldsymbol{P}_{\boldsymbol{e}}$ should also be much lower to counteract with its scale effect. While the original signal number level is relatively low (num=10), $\boldsymbol{P}_{\text {dam }}$ and $\boldsymbol{P}_{\boldsymbol{e m}}$ need to be both high to reach the general trend in experimental data. 
As $\boldsymbol{P}_{d a m}$ and $\boldsymbol{P}_{\boldsymbol{e m}}$ differ from each set of parameters, there should be differences on the temporal characteristics. By referring to the experimental data ${ }^{[5]}$, it could be verified that the damage in total reached a peak when 3 days after the irradiation of X-ray. In the simulation, when the original signal number is 30, the low level of $\boldsymbol{P}_{\text {dam }}$ and $\boldsymbol{P}_{\boldsymbol{e}}$ makes the signals annihilated approximately 2 days after the irradiation. It also indicates that the damage peak would probably come out 2 days after irradiation or even earlier. While in the other two sets of parameters, since $\boldsymbol{P}_{\boldsymbol{e}}$ is relatively high, signals keep existing for 3 days or a little longer, which renders the prediction of these two sets more reasonable.

After having modulated and compared the parameters, the simulation of other experiment was made with parameters obtained to testify the rationality of this model. In the previous Monte Carlo model ${ }^{[8]}$, the basic experimental condition was that 841 cells were settled on each definite spot in a rectangular dish and the distance between two neighboring cells group was $0.35 \mathrm{~mm}$, with the central cell irradiated. The results of the previous model without "shaking effect" are shown in figure $3 \mathrm{a}$.

The previous work generated contradictory results to the experimental data ${ }^{[5]}$. On the contrary, our present model resolved the problem. As shown in the figure $3 \mathrm{~b}$, the simulation results agree well with the experimental data shown in the figure $2 . b$ of the ref. ${ }^{[5]}$. The characteristic of the simulation results is that the damaged cells are located rather randomly in the dish than centralized in the center. When employing the assumption that the signal will be absorbed even if it fails to cause damages, almost no signal is able to escape from the center of the dish, even in the situation where $\boldsymbol{P}_{\text {dam }}$ 
is quite low. Signals are all absorbed by the cells in the centric area. However, by assuming that the signal will continue moving in the medium instead of being absorbed, it is capable of spreading to a longer distance, leading to the result that the damaged cells distribute comparatively separately in the dish.

After all, the perfect agreement between the experimental data and the present simulation results indicates a multi-stage amplification of signal transmission in RIBE. And TGF- $\beta 1$ is a good candidate of signaling molecule in the medium. The simulation also demonstrates that RIBE is triggered by both the primary signals from the irradiated cells and the re-emitted signals from the bystander cells. Further investigation is going on to study the dependence of the value of $\boldsymbol{P}_{\boldsymbol{d a m}}, \boldsymbol{P}_{\boldsymbol{e m}}$ and lifespan on the different experimental conditions such as different endpoints, cells and nourishing conditions.

\section{CONCLUSION}

In this paper, a Monte Carlo model is developed to study the mechanisms of bystander effect in cells sparsely populated conditions. The model, based on the experiment where the cells are sparsely distributed in a round dish, focuses mainly on the spatial characteristics. The simulation results successfully reach the agreement with the previous experiments. The comparison of the simulation and experimental results indicates the feasibility of the model and the validity of some vital mechanisms assumed 


\section{Acknowledgements}

$\mathrm{Wu}$ and Wang want to thank Gray Cancer Institute for hosting them for three months and providing of microbeam for cell irradiation. The authors appreciate the financial support from the National Science Foundation of China (Grant No. 10435020, 10225526). Xia and Liu thank the support from the President's Undergraduate Research Fellowship (PURF), Peking University.

\section{Reference}

[1] W.F. Morgan, Radiat. Res. 159 (2003a) 567-580.

[2] W.F. Morgan, Radiat. Res. 159 (2003b) 581-596.

[3] T.K. Hei, Mol Carcinog. 45 (2006) 455-460.

[4] B. Hu, L. Wu, W. Han, L. Zhang, S. Chen, A. Xu, T. K. Hei and Z. Yu, Carcinogenesis 27 (2006) 245-251.

[5] K.M. Prise, O.V. Belyakov, M. Folkard and B.D. Michael, Int. J. Radiat. Biol. 74 (1998) 793-798.

[6] D. J. Brenner, J. B. Little, and R. K. Sachs, Radiat. Res. 155 (2001) 402-408.

[7] H. Nikjoo, and I. K. Khvostunov, Int. J. Radiat. Biol. 79 (2003) 43-52.

[8] F. Ballarini, D. Alloni, A. Facoetti, A. Mairani, R. Nano and A. Ottolenghi, Radiat. Prot. Dosim. 122 (2006) 244-251.

[9] C. Shao, M. Folkard and K.M. Prise, Oncogene 27 (2008) 434-440.

[10] L. Wu, Y. Wang. Private communication.

[11] K. Jacobson and J. Wojcieszyn, Proc. Natl. Acad. Sci. USA 81 (1984) 6747-6751.

[12] E.I. Azzam, S.M. de Toledo, J. B. Little, Proc. Natl. Acad. Sci. USA 98 (2001) 473-478. 
[13] C. Mothersill and C. B. Seymour, Int. J. Radiat. Biol. 71 (1997) 421-427.

Table 1. Modulation results of parameters at different levels of the original signal number.

\begin{tabular}{|c|c|c|c|}
\hline Original signal number & $\mathrm{P}_{\mathrm{dam}}$ & $\mathrm{P}_{\mathrm{em}}$ & 1 ifetime \\
\hline 10 & 0.004 & 0.9 & $1.5 \mathrm{~d}$ \\
\hline 20 & 0.0018 & 0.95 & $1.5 \mathrm{~d}$ \\
\hline 30 & 0.002 & 0.15 & $1.5 \mathrm{~d}$ \\
\hline
\end{tabular}




\section{Captions:}

Figure 1. One sample dish in the experiment where 130 cells were located randomly in the dish and the centric cell was directly irradiated with $1 \mathrm{~Gy} \mathrm{X}$-rays.

Figure 2. Simulation results of our previous experiment. The simulated dish has a radius of $6000 \mu \mathrm{m}$. In every area, from the left to the right, the bars represent the ratio of damaged cells resulted from the experiment, simulation with 10,20 and 30 original signals. Other detailed parameter values are shown in the table 1.

Figure 3a. Top view of the 841-cell grid with an example of damage pattern obtained by the previous Monte Carlo model simulating the release of $\mathrm{N}$ signaling molecules by the irradiated and bystander cells, with $N=100, \boldsymbol{P}_{\text {dam }}=0.04$, $\boldsymbol{P}_{e m}=0.5$.

Figure $3 \mathrm{~b}$. Top view of the 841-cell grid with an example of damage pattern obtained by this present model simulating the release of $\mathrm{N}$ signaling molecules by the irradiated cell, with $\mathrm{N}=20, \boldsymbol{P}_{\boldsymbol{d a m}}=0.0018, \boldsymbol{P}_{\boldsymbol{e m}}=0.95$ and the newly proposed parameter lifespan $=1.5$ days in real time. 


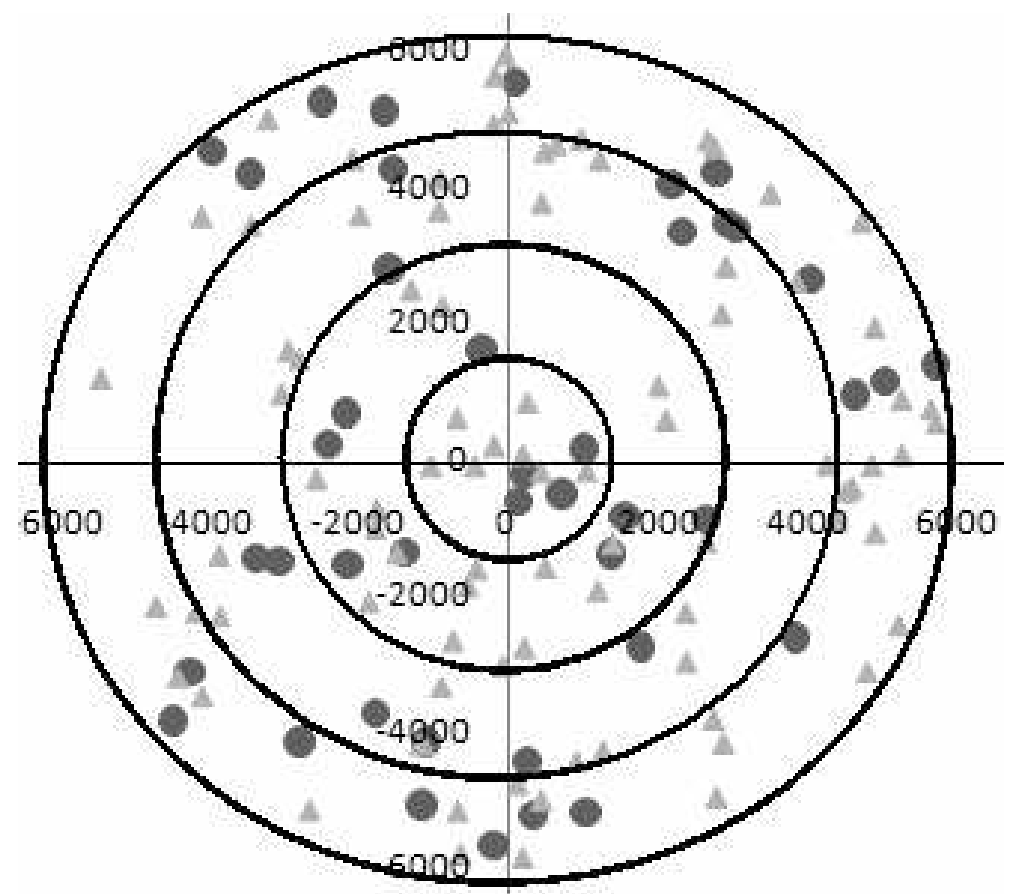

Figure 1

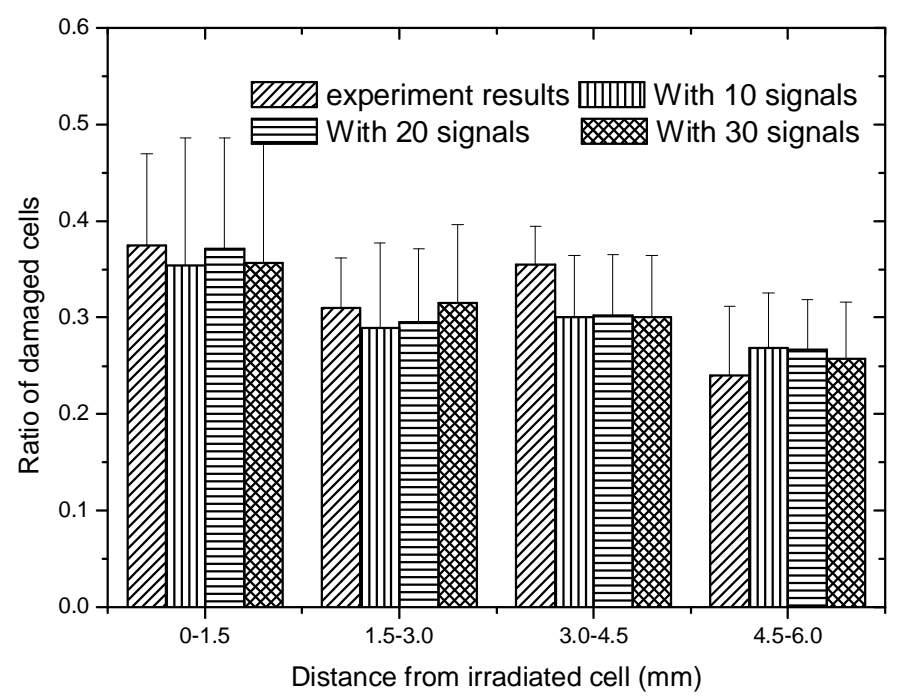

Figure 2 


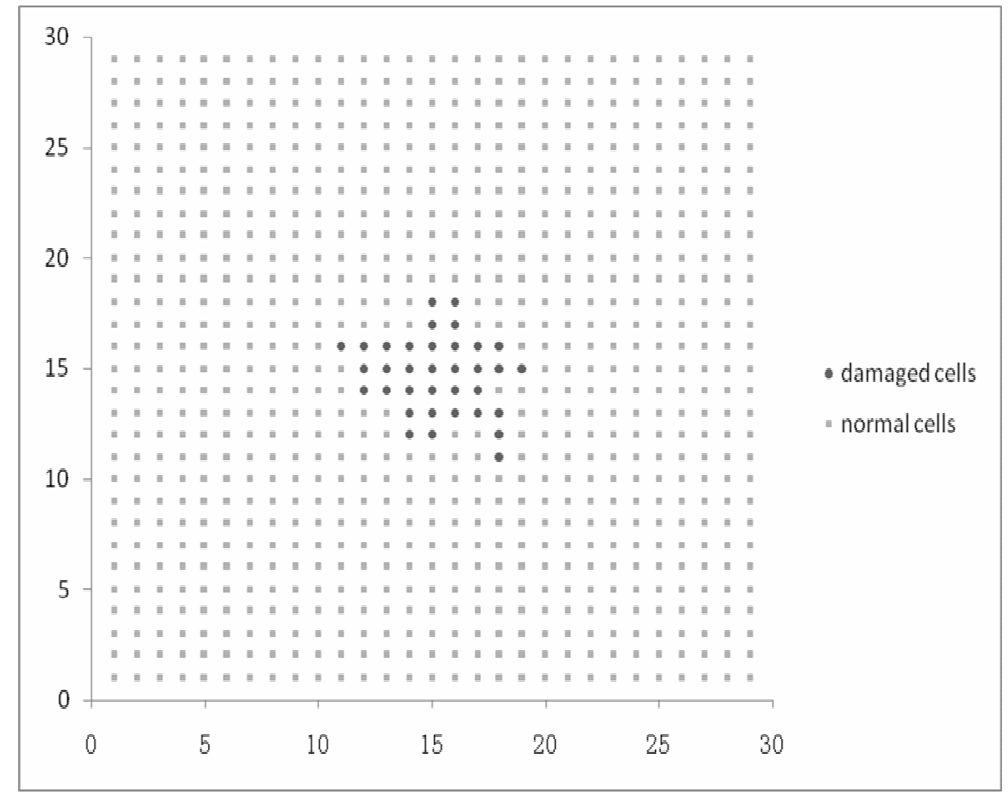

Figure 3a

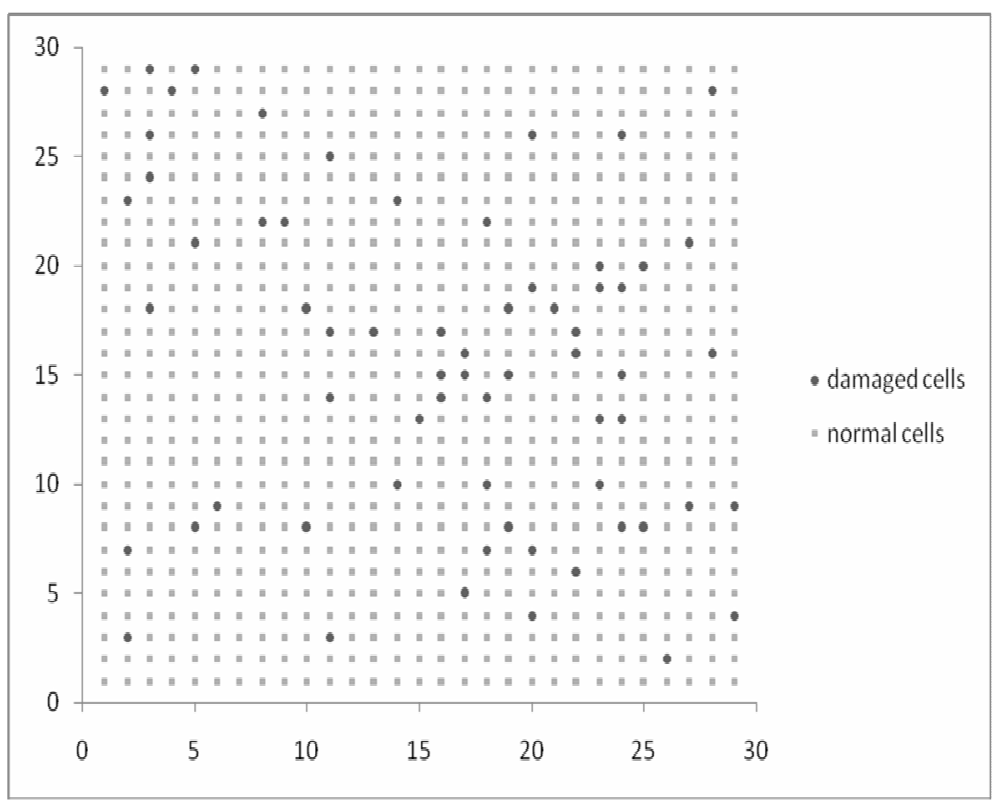

Figure $3 b$ 